\title{
Energy Optimization in Wireless Sensor Network through Natural Science Computing: A Survey
}

\author{
Sanjeev Wagh ${ }^{1}$, Ramjee Prasad ${ }^{2}$ \\ CTiF - Centre for TeleInFrastruktur, Aalborg University, Aalborg, \\ Denmark E-mail: $\left\{{ }^{1}\right.$ wagh, ${ }^{2}$ prasad $\} @ e s . a a u . d k$
}

Received: June 17, 2013; Accepted: September 27, 2013

\begin{abstract}
Nature-inspired computational algorithms are attracting engineering research for artificial intelligence solutions. The Natural science modelling for designing intelligent artefacts gives good results which reliably execute in the system. Some time it is expensive in terms of space and time to create reliable artefacts with conventional process that design the elements like intelligence, controlling operations, process optimization, threat and security, design of the future ideas, and so on. The paper addresses the natural science processes and solution directions. It integrates contrasting techniques of Genetic systems, Neural System, Immune systems and Cellular automata inspired from human organs to give solutions for energy conservation problems in Wireless sensor network domain. The non-human aspects of natural science like, swarm optimization, social insects like ant honey bees, wasps, termites and many more are also discussed to solve many real-world problems. The paper gives insights into the creation of innovative improvements over algorithm performance, potential applications on various practical tasks, and combination of different techniques for energy optimization in wireless sensor networks.
\end{abstract}

Keywords: Energy optimization, Natural systems, Human organ components, swarms, Optimization levels, etc.

Journal of Green Engineering, Vol. 3, 383-402.

doi: 10.13052/jge1904-4720.342

(C) 2013 River Publishers. All rights reserved. 


\section{Introduction}

The purpose of any Research is to provide easy and comfortable living to present and next generation of every Human life and other living things with the help of technology. When researcher confirms that the conventional approaches look inefficient for the expected results, they search for alternative dimensions. Most inspired direction for research now-a-days is Natural science and its creative miracle- self behavior patterns.

The Information Technology has made a great change in the human societies in terms of transportation, industrial production, administration, technological advances, entertainments and many more. There are several problems that are hard to solve by conventional process. The conventional process has certain limitations like structure oriented bindings, partially predictable and computable in limited time and space. Some hard problems like travelling sales person, chess playing, intelligent human computer interaction, natural language understanding, robotics, business and planning cannot justify by conventional method. Hence the non-conventional process like natural science is always inspiring direction for solving the technical problems. There are many fields of application like Process optimization, Telecommunication, Entertainment; etc can be solved affordably by using the analogy of natural science theme.

Natural Science inspired design based on understanding deep and accurate analogies, concepts between human and natural systems. The design principles useful to a human problem must be derived from analyzing a similar problem faced in the natural world. Still it is not fully understood how experts in engineering or natural science field will go about searching evolutionary adaptation as a source for design inspiration in great depth. The technical applications systems are required to understand the problems and social processes underlying naturally inspired design as per Figure 1 . The conclusion of these application studies are computational models and tools that support the transfer of natural knowledge to engineering domains and educational strategies that will motivate the Engineers and Environmentalist to work on interdisciplinary framework.

The Natural Science inspired algorithms solved the problems according to the nature's principles - self behaviour system. To solve the technical problems one should understand the nature's rules, principles and mechanism. The Natural Science system contains multiple dimensions and space and have many elements connected in complex ways. Examples include networks of self-regulating circulatory vessels, social insect colonies, or ecological 


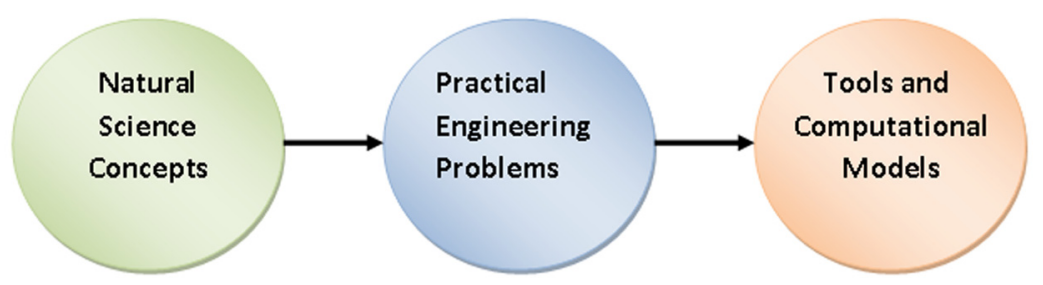

Figure 1 Process of Natural Science to Practical computational solutions

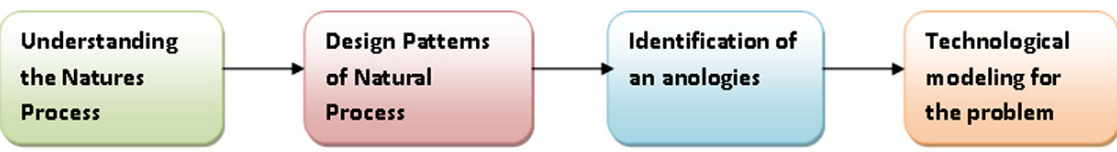

Figure 2 The process of designing intelligent system through Nature inspired system

communities. These systems often exhibit surprising complexity and perform well under extreme conditions, even though individual interactions may be based on simple rules like bee colonies. In some cases the natural ecosystem resist the abnormal behaviour caused due to individual elements, may be get removed or added to the system. Most natural systems function to exchange information and contents by verifying the properties. These systems provide strategies for more reliable, efficient and sustainable solutions for transportation, energy management like problem, which will be more secure and robust. Also provide strategies for more adaptive behaviour of groups example movement rules, task allocation, etc with a minimal number of simple rules and slight changes in organizational hierarchy.

The human organs sense physical physiological response like motion, sound or light with the help of structures and processing schemes. The strategies are often different from that employed in artificial systems, because humans are visually oriented. The survivals of human are based on their organism's ability to gather information efficiently. Organisms perform these tasks under conditions of limited processing power or materials. Studying non human natural elements like social insects sensation therefore give solution to novel sensors, or develop sensors that efficiently gather particular information for a certain task in a specific environment. The limits on neural processing machinery and sensory structures make animal strategies particularly useful for autonomous systems. The other elements like animals must also frequently communicate without exposing themselves to predators or other dangers, and provide insights in how to design private communication channels. 


\section{State of an Art - Why Natural Systems?}

In the future Internet the critical issues will be concern to the interconnection and integration of the physical world and the virtual world. The challenges will be addition of the high volume of content together with new emerging and mission critical applications, which will be stressing the Internet to high degree. The area of research where we can concentrate to cope with future internet is with scalability, mobility and variety. We need to adjust with growing number of network devices, end users attached to the network and their network planning's. The mobility of users and also network nodes always require reliable connectivity under different traffic conditions.

To advance the state of the art that the end hosts must be equipped with adaptability to the current network condition for finding communicating peers with congestion controls. Also at the same time, network control should satisfy the end host requirements in a real-time. Since the network is becoming complex and large-scaled, it becomes more difficult to utilize a conventional well-managed approach for unpredictable conditions [29].

The proposed approaches, target the new network control methods in order to tackle current problems or to improve existing proposals with certain assumptions. The input assumes processing capability, topology of the network, traffic characteristics and required QoS. The proposed system consider the throughput and/or packet delays as the performance average metrics but the validation of using those metrics is based on the stable system. The future network will be extreme dynamic due to the frequent change in topologies of device, contents and traffic behaviors. Hence in the future networks, we have to seriously consider the related metrics in order to justify the quality level of the network i.e. manageability, availability, reliability, adaptability, dependability, sustainability, re-configurability, and others issues.

The State of art proposal suggests the application of biological agent's characteristics which is based on the RAIN properties [30]. RAIN: Recognize characteristics, Avoid by protection, Isolate the hazards and Notify the appropriate resources and authorities when responding to event. It gives surprising results for the design of intelligent artefacts that are sufficient to execute in target system. It is difficult to module the intelligence that design the elements like intelligence, controlling operations, process optimization, threat and security, design of the future ideas, and so on. The proposal clarify these needs and integrates contrasting techniques of Genetic systems, Neural System, Immune systems and Cellular automata inspired from human organs to give solutions for maximum problems in wireless sensor network domain. The 
non-human aspects of natural science like, swarm optimization, social insects like ant honey bees, wasps, termites and many more are discussed to solve many real-world problems. The theme presented in the paper give insights into the creation of innovative improvements over algorithm performance, potential applications on various practical tasks, and combination of different techniques.

\section{Intelligent Solutions for WSN applications}

There are several research attributes and parameters in wireless sensor networks that can be solved using natural process like a decentralized algorithm between sensors which should work for everything, using computational abilities i.e. hardware characteristics of sensors, an algorithm can be designed for faster solutions and better results. More constraints can be added like continuous variations in links status, mobility of sensor nodes, multihop operation through the cluster head or normal sensor nodes, multiple changing links, three dimensional environment, etc in wireless sensor networks.

\subsection{The Human organ components for WSN application}

The components of natural science i.e. human body organ are selected for modelling solutions to wireless sensor networks concern to energy optimization by inheriting their working strategy as shown in Table 1 .

The distinguished features are passed from parents to offspring through gene transmission. Genes are located on chromosomes and consist of DNA. They are passed from parent to offspring through reproduction. [1] Azad nazi and et.l. designed the biological gene structure to design wireless sensor networks. The concept Gene Regulatory Network (GRN) of the real living organisms which gives information about intrinsic properties of adaptation and robustness which have used for creating dynamic network environments. The researcher use the strength of the robust GRN topology as a template to design communication structure of the WSN.

The nervous system of human consists of the brain, spinal cord, and a complex network of neurons. This system is responsible for sending, receiving, and interpreting information from all parts of the body. The nervous system monitors and coordinates internal organ function and responds to changes in the external environment. [2] H. He, Z. Zhu et. 1. proposed a neural network model to find the minimum Weakly Connected Dominating Set in a wireless sensor network. The proposal presents a directed convergence and proves 
Table 1 Nature science inspiration from human organ functions

\begin{tabular}{|c|c|c|c|}
\hline Sr. no. & $\begin{array}{l}\text { Components of } \\
\text { Natural Science }\end{array}$ & $\begin{array}{l}\text { Mechanism } \\
\text { based on }\end{array}$ & WSN Applications \\
\hline 1. & Genetic System & The Genes & $\begin{array}{l}\text { Optimum time for topology transi- } \\
\text { tions Intelligent Routings in WSN } \\
\text { Intelligent Clustering in WSN }\end{array}$ \\
\hline 2. & Neural Systems & $\begin{array}{l}\text { The brain, spinal } \\
\text { cord \& neurons. }\end{array}$ & $\begin{array}{l}\text { Intelligent data acquisition for analy- } \\
\text { sis Intelligent routing with change in } \\
\text { topology Integration of multiple type } \\
\text { of data and knowledge }\end{array}$ \\
\hline 3. & $\begin{array}{l}\text { Cellular } \\
\text { Automata }\end{array}$ & The cells grid & $\begin{array}{l}\text { Dynamic network topology manage- } \\
\text { ment Multipath data transmission } \\
\text { management Energy overhead. }\end{array}$ \\
\hline 4. & Immune system & $\begin{array}{l}\text { The protection } \\
\text { mechanism }\end{array}$ & $\begin{array}{l}\text { Intelligent Routing is based on opti- } \\
\text { mized data Optimum time for topol- } \\
\text { ogy transitions }\end{array}$ \\
\hline
\end{tabular}

the scalability of the neural network model by testing it on a range of sized graphs and on a range of transmission radius. Another [3,4] intelligent agent based scheme on a neural network topology which do significant reduction in the energy resource consumption required for training and operation of nodes without compromising its change detection performance. The Azzam I. M. et.l. use neural network approach for recurrent neural network to model sensor nodes and their dynamics. The topology is updated based on neighbouring sensors information.

A cellular automaton consists of a regular grid of cells, each in one of a finite number of states, such as on and off. The grid can be in any finite number of dimensions. For each cell, a set of cells called its neighbourhood is defined relative to the specified cell. An initial state is selected by assigning a state for each cell. A new generation is created, according to some fixed rule that determines the new state of each cell in terms of the current state of the cell and the states of the cells in its neighbourhood. Typically, the rule for updating the state of cells is the same for each cell and does not change over time, and is applied to the whole grid simultaneously, though exceptions are known, such as the probabilistic cellular automata and asynchronous cellular automaton. The synthetic cellular automation provided by Stavros A. \& et. 1. [5] is decentralized computing model provide complex computation in wireless sensor network using local information. The proposal assumes Moors neighbourhoodof eight surrounding nodes. The method [7] combines local evolutionary design rules with finite element analysis. The design rule 
incorporated for minimizing error between local mechanical signal and its optimum price by different control strategies.

Within an organism the system that protects against disease are known to the immune system, it's a system of biological structures and processes within. An immune system is used to detects a wide variety of agents from viruses and parasitic worms and distinguish them from the organism's own healthy tissue for functioning organ properly. The heuristics method [6] inspired from immune system where antibodies are decided as solutions to the problem and antigen is the problem. It tries to justify the multi-objective minimum energy network connectivity problems which optimize the energy. In another model [8] the range for selected nodes is decided and affinity rate is evaluated. Based on this evaluation, selected node declared as memory cell and the links of other nodes are mutated. The process continues until the number of iterations exceeds to threshold rate or the affinity rate of all nodes become good enough than threshold rate.

\subsection{The Nonhuman living components for WSN application}

A group of insects or other small organisms especially when in motion intelligently exchange the information about paths and directions. A group of bees with a queen bee migrate to establish the new colony intelligently. There is several real life problems can be optimized intelligently using the behavioural study based on organisms like social insects. The Nature Science inspiration from nonhuman living functions for wireless sensor network applications are integrated in Table 2.

The Swarm is combinebehaviour of social insects group in nature, such as ant colonies, honey bees and bird flocks $[9,10]$. These agents interacts together with certain behavioural patterns to cooperative and achieve tasks necessary for their survival. They have relatively limited capabilities on their own as individual. Due to the amazing grouping efficiency of the natural swarm, biologists and natural scientists inspired to have a study of these behaviours of social insects give efficient solution to practical problems. In the late-80s, computer scientists proposed the scientific insights of these natural swarm systems to the field of Artificial Intelligence. The Swarm Intelligence was first introduced by G Beni and J Wang in their proposal of global optimization framework, a set of algorithms for controlling robotic swarm in 1989 [11].

The interaction among swarm as individuals can be either direct or indirect. The different societies of swarm are shown in Figure 4. The direct interactions 
Table 2 Nature science inspiration from swarming functions

\begin{tabular}{|c|c|c|c|}
\hline Sr. No. & $\begin{array}{l}\text { Components } \\
\text { of Natural } \\
\text { Science }\end{array}$ & Concept Inheritance & WSN Applications \\
\hline 1. & $\begin{array}{l}\text { The } \\
\text { Swarm }\end{array}$ & $\begin{array}{l}\text { group foraging of social } \\
\text { insects cooperative } \\
\text { transportation Division of } \\
\text { labour \& building nests. } \\
\text { collective sorting and clus- } \\
\text { tering }\end{array}$ & $\begin{array}{l}\text { distributed system of interacting } \\
\text { autonomous agents Performance } \\
\text { enhancement, process optimization } \\
\text { and robustness. self-organized con- } \\
\text { trol and cooperation in decentralized } \\
\text { environment division of process and } \\
\text { distributed task allocation }\end{array}$ \\
\hline 2. & $\begin{array}{l}\text { The } \\
\text { Honey } \\
\text { Bees }\end{array}$ & $\begin{array}{l}\text { Efficiently combine repli- } \\
\text { cation and evasion to allow } \\
\text { network to continue deliv- } \\
\text { ering data for a long time } \\
\text { during a jamming attack. }\end{array}$ & $\begin{array}{l}\text { An energy-aware defense frame- } \\
\text { work against base-station jamming } \\
\text { attack in WSNs. }\end{array}$ \\
\hline 3. & The Ants & $\begin{array}{l}\text { Control is fully distributed } \\
\text { among a number of indi- } \\
\text { viduals Communications } \\
\text { among the individuals hap- } \\
\text { pening a localized way. } \\
\text { The overall response of } \\
\text { the system is quit robust } \\
\text { and Adaptive with respect } \\
\text { to changes in the environ- } \\
\text { ment }\end{array}$ & $\begin{array}{l}\text { Allocation of the computing } \\
\text { resources to number of cluster units } \\
\text { The Control can be fully distributed } \\
\text { among a number of clusters The } \\
\text { cluster interacts in simple and } \\
\text { localized ways. }\end{array}$ \\
\hline 4. & $\begin{array}{l}\text { The } \\
\text { Wasps }\end{array}$ & $\begin{array}{l}\text { A colony of individuals } \\
\text { working together for the } \\
\text { survival of the colony } \\
\text { Coordinate their behavior } \\
\text { in order to build such com- } \\
\text { plex nest structure }\end{array}$ & $\begin{array}{l}\text { Applications of self-assembling } \\
\text { robots Cluster formation in sensors } \\
\text { and evolutionary design to architec- } \\
\text { ture in WSN }\end{array}$ \\
\hline 5. & $\begin{array}{l}\text { The } \\
\text { Termites }\end{array}$ & $\begin{array}{l}\text { Termites move from a } \\
\text { non-coordinated to a coor- } \\
\text { dinated phase only if their } \\
\text { density is higher than a } \\
\text { threshold value. }\end{array}$ & $\begin{array}{l}\text { To balance the network traffic load } \\
\text { and prolong the network lifetime } \\
\text { without performance degradation. }\end{array}$ \\
\hline
\end{tabular}

are through visual or audio contact, such as the waggle dance of honey bees. Indirect interaction occurs when one individual changes the environment and the other individuals respond to the new environment, such as the pheromone trails of ants that they deposit on their way to search for food sources. This indirect type of interaction is referred to as stigmergy, which essentially means communication through the environment [12]. 


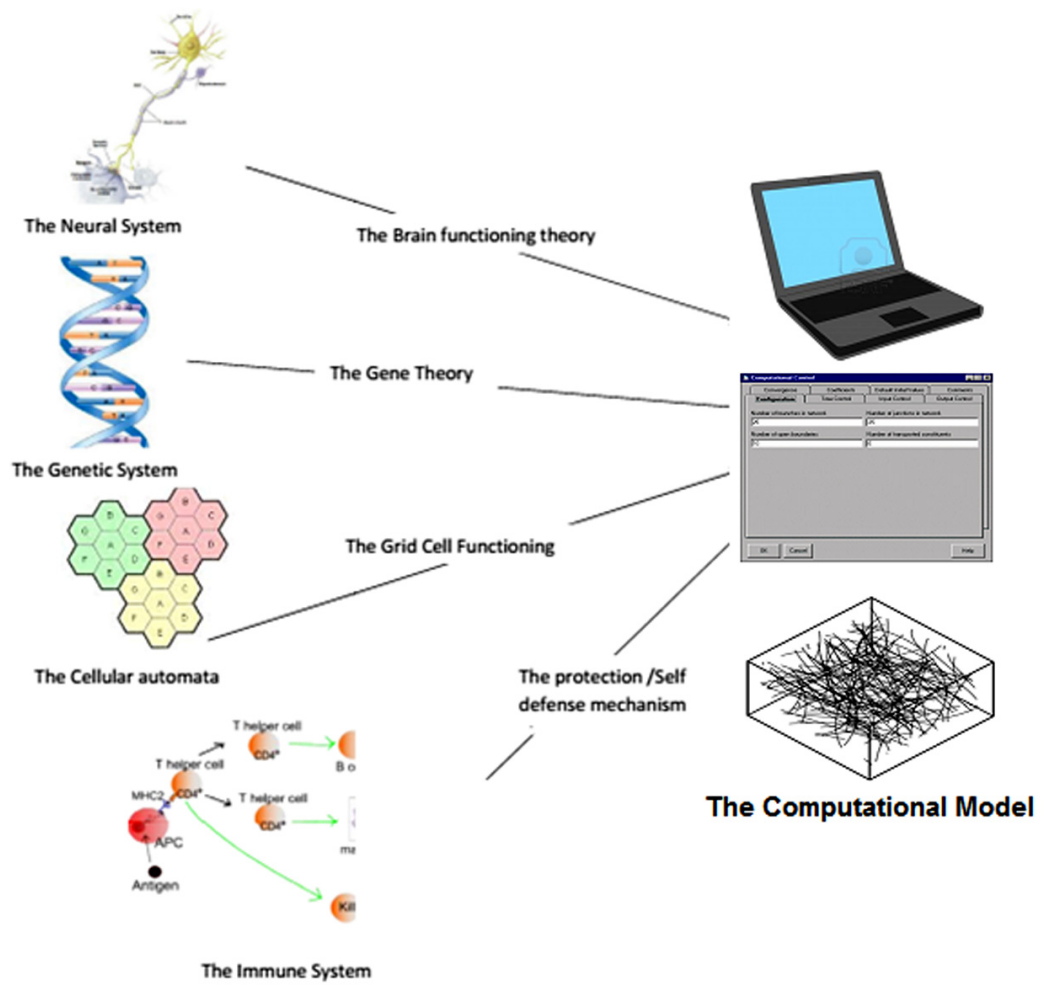

Figure 3 The Intelligent solution using Natural Science components

The existing problems in communication networks can be efficiently solved using the Swarm intelligence. The solution to the problems like distributed system for interacting autonomous agents, performance optimization, robustness, self-organized control and decentralized cooperation can be possible for applications in wireless sensor networks. The other applications like indirect interactions, division of tasks and distributed task allocation in network operating system can effectively works.

The study of swarm intelligence is providing insights that can help researchers to manage complex systems, for number of the applications in real life. The ants are considered as grand masters in search and exploitation. A single ant or bee isn't smart for the solutions, but their colonies are smart. The mechanism of an ant can be inherited for solution as cooperation and division of labour, adaptive task allocation, work stimulation by cultivation and the use pheromones the chemical substance. Ant colony optimization algorithm 


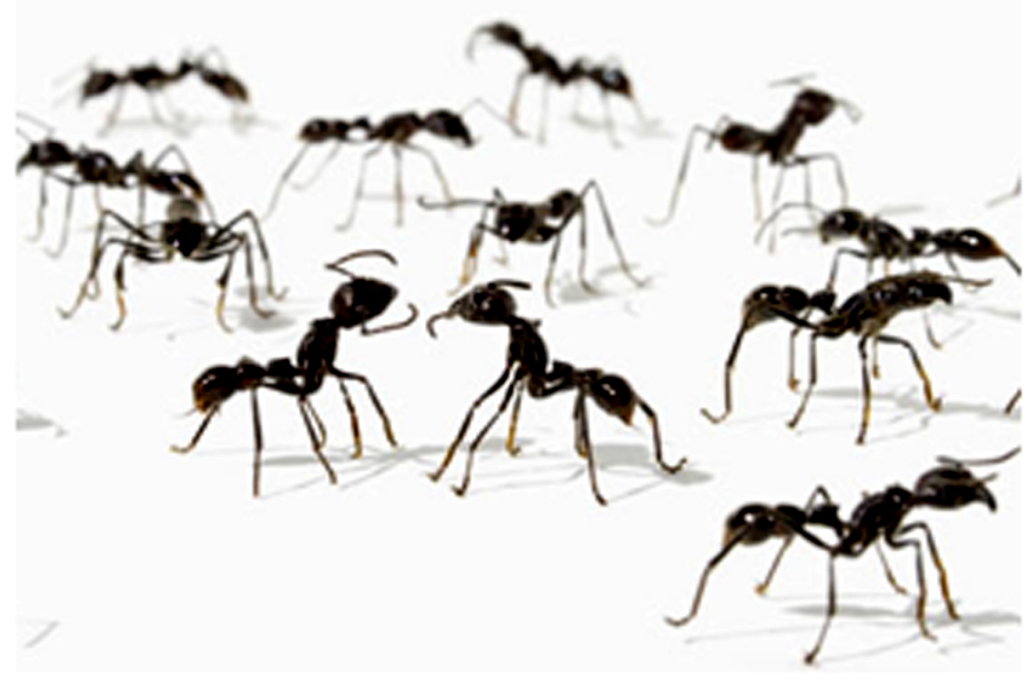

Figure 4 Type of Swarms

used effectively for solving discrete optimization problems, inspired from the behaviours of an ants.

The conceptual model in [14] considers pheromone update rule, probabilistic transition rule and heuristic desirability. Probabilistic transition rule determine the probability of choosing an edge i.e. an ant's path by considering the existing pheromone level and its heuristic desirability. Pheromone updating rule specifies how the concentration of pheromone on the edges are modified by both depositing and evaporating procedure. Besides giving solution to search possible paths, using pheromone level and desirability is also considered for the inverse of the edge length, by which the ants choose a shorter path. The techniques [14] used by antcolonies have been studied to model distributed and fault-tolerant systems for solving problems. This area of studies of ant's locomotion is called biomimetics which led to, search engines that make use of foraging trails for fault-tolerant storage and networking algorithms.

The methods proposed in [13] maximize the path availability and minimize travel time of packets using ant colony optimization algorithms of Swarm intelligence techniques. It offers a good balance between selection of fast paths and a better use of network resources. Another example of swarm species are honey bees. When a honey bee swarm comes from a hive they fly to limited distance at start. Some few meters from the hive, they gather in a tree or on a 
branch. There, they group around the queen and select some 20-50 scout bees to send for finding a suitable new nest location. The scout bees are the most experienced searcher in the group. An individual scout returning to the group and broadcast a location she found. She uses the waggle dance to indicate direction and distance to others in the group to convince other members to check out the location she found. The others may take off, check out the proposed site and promote the site further upon their return. Several different sites may be promoted by different members at first. After several hours and sometimes days, slowly a favourite location comes from this decision making process. When all members agree on a final location the whole group takes off and flies to it. This collective decision making process is remarkably successful in identifying the most suitable new nest site and keeping the swarm intact.

The logic inspired from the honeybee swarm is modelled in [16]. The proposed method is based on natural bees' communication. The system confirms the proof of work efficiently in either a homogenous or a densely populated network. The protocol is compared with DIR routing protocol for finding shortest path. Both algorithms use the notion of angles to choose the shortest path by selecting the smallest angle in each hop toward the final destination. Another approach in [17] uses the Queen-bee algorithm to create energy efficient clusters in wireless sensor networks. The author justifies that the clustering by the QB algorithm decreases the energy consumption with regard to the other existing algorithms and increases the lifetime of the network.

The behavior of social insects that live in colonies, such as ants, bees, wasps and termites, has always been a source of fascination for naturalists and artists. Individual insects seem to do their own thing without any central control, yet the colony as a whole behaves in a highly coordinated manner. The combine effort of such behaviors can solve highly complex problems. The stimulus-response mechanism of wasp colony is introduced in [20] to determine the probability of response to the task invitation to avoid consuming extra energy. Simulation results show that our model efficiently reduces energy consumption and network traffic, decreases the number of dead nodes, and prolongs the lifetime of the networks.

The Termites are another swarm species works with three types of agents: reproductive, soldiers and worker based system. In reproduction phase male reproductive king and the female reproductive queen are the primary source of pheromones which is useful in colon integration, and these are thought to be spread through shared feeding in termite colonies. The soldier's agent classifies as forward soldiers and backward soldiers depending upon the direction 
in which they travel. The third agent undertakes the labors of foraging. The workers feed the other members of the colony with substances derived from the digestion of plant material, either from the mouth or anus. The proposal [19] explores termite colony optimization Meta heuristic termed Termite-hill. The proposed algorithm efficiently relay all the traffics destined to the target, and also balance the network energy and increase the network lifetime without performance degradation.

\section{Intelligent Solutions for WSN applications}

The energy optimization techniques can be classified with static or dynamic strategy. In static optimization, the energy optimization is planned at deployment time and remained fixed for lifetime of network. The strategy is useful

in case of stable and foretells system. The dynamic strategy is more flexible to provide solution supporting with response to change in requirements and environment. We can target at the different levels of OSI reference model for energy optimization. Table 3 shows the parameters of research at different layers and nature inspired methods for energy optimization.

\subsection{Physical level parameters}

The wireless sensor network utilizes battery operated nodes and consumes energy in transferring data from one node to other target node. The routing of data takes place through several nodes and not directly. The most of the energy get wasted due to noisy channels and other physical parameters. The scheme proposed by Matthew Holland and et.1. [22] suggests energy-efficient transmission of data over a noisy channel, focusing on the setting of physical layer parameters. The metric module of the energy per successfully received bit, which specifies the expected energy required to transmit a bit successfully over a particular distance. By minimizing this metric, different modulation scheme can be tested for energy optimal relay distance and the optimal transmit energy as a function of channel noise level and path loss exponent. The ready components such as a power unit, storage unit, sensing unit, processing unit, of sensor nodes provide optimization opportunities for tunable parameters like processor voltage and frequency, sensing frequency, duty cycle, etc., whose values can be specialized to meet varying application requirements. The effective optimization can be possible with the neural system [2-4] and the swarm - an ants [14] analogy. 


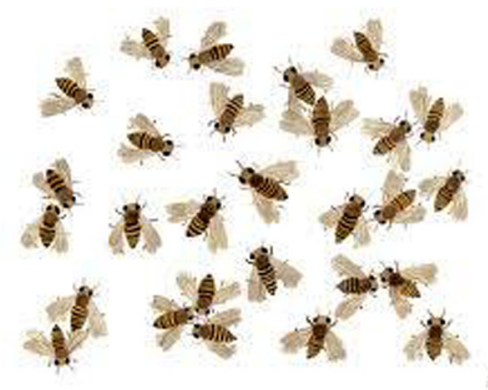

Figure 5 The Intelligent solution using swarm components

\subsection{Data link level parameters}

The wireless sensor network infrastructure is dynamic in nature due to onoff nodes status, self-organization and failure recovery. To meet application requirements, WSN designers can tune MAC layer protocol parameters i.e. channel access schedule, message size, duty cycle, and receiver power off, etc [21].

The wireless channel slots can be adjusted to optimize throughput while maintaining the traffic load balance between sensor nodes and packets delivery to the sink node from all the senders can be measured by a fairness index for maintenance. The transceiver operating modes and duty cycle for reduced power and/or energy consumption can be adopted by MAC layer protocols. MAC layer protocols adjust duty cycle for power/energy optimization includes Power Aware Multi-Access with Signalling. The energy optimization issues can effectively handle using human immune system [6,8] and the Swarms like wasps and an ants $[20,14]$ concepts.

\subsection{Network level parameters}

The energy efficiency, robustness and scalability can be achieved through data dissemination and routing protocols in Network layer. The optimization can be achieved through routing protocols by adjustment in transmission power, routing strategies, and leverage either single-hop or multi-hop routing. The query disseminations and data forwarding requires routing layer optimizations. The applications like defence system for monitoring enemy troops or forest fire detection requires the real sensed data delivery so, the Routing 


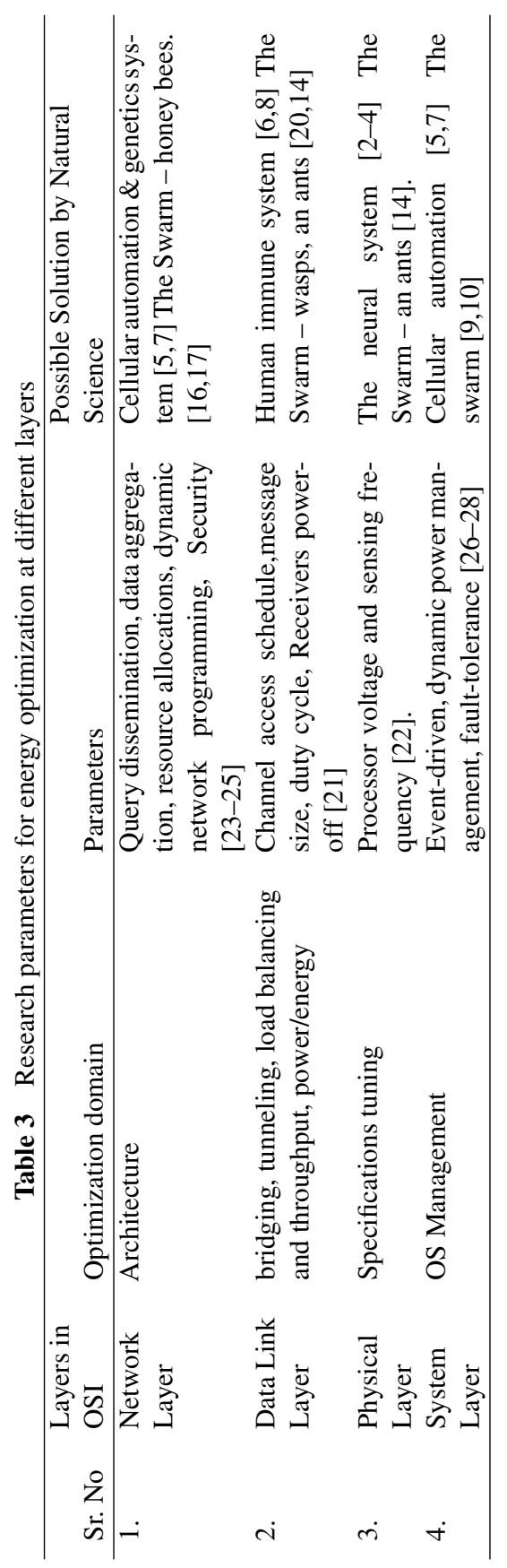


protocols consider the timing constraints for real-time requirements. The Realtime Architecture and Protocol (RAP) [23] and a stateless protocol for realtime communication in sensor networks (SPEED) [24] achieve the need but still there are lots of scopes for research. The network topology and radio transmission power can be adjusted by routing protocols. The Low-Energy Adaptive Clustering Hierarchy (LEACH) [25] optimizes the network topology for reduced energy consumption by adjusting the radio's transmission power. LEACH uses a hybrid single-hop and multi-hop communication model in which the sensor nodes use multi-hop communication to transmit data reports to a cluster head. The application of nature science procedures like cellular automation, genetics system [5,7] and the Swarm - honey bees [16,17] can give effective solution for energy conservation

\subsection{System level parameters}

The sensor node operates between single-application devices without OS and share general-purpose devices with resources with the help of embedded OSs. A sensor node's OS manages processor, radio, I/O buses, and Flash memory, and provides hardware abstraction to application software, task coordination, power management, and networking services [21]. By controlling sensing and actuation activity sensor nodes respond to events and as sensor nodes are event-driven, optimization of the OS for event handling is possible to optimum energy utilization. WSN Oss optimized for event handling in TinyOS [26]. The hardware components like power management in sensor node's OS can control to optimize power consumption. The idea [27] explores that switching the sensor node to a sleep state when idle for energy conservation; however, transition to sleep state has the overhead of storing the processor state and requires a finite amount of wakeup time [27]. Once deployed, maintenance and repair of sensor nodes not possible hence it require fault-tolerant mechanisms for reliable operation. The multithreaded OS [28] provides fault-tolerant isolation between applications by stopping a blocking task to prevent the execution of other tasks. The energy optimization issues can effectively handle using Cellular automation [5,7] and the swarm $[9,10]$ concepts.

\section{Research Scopes And Future Directions}

Future research trends in WSN dynamic optimizations include the investigation of algorithms suitable for sensor nodes with constrained resources and 
energy. There are number of challenges in existing networks like Exchange of data in large-scale heterogeneous networks and their elements, integration and interaction adaptation of uncertain information effectively and Service adaptation in the dynamic system environment The stated problems can be effectively minimize or absolute using with natural science techniques inspired from self organization behavioural patterns of human and non-human system.

We particularly focus on the nature inspired energy optimization algorithms which can be applied in design, planning, and control problems concern to energy optimization in wireless sensor networks. We roughly group those methods into two categories, one is based on human organ function i.e. genetic system (GA), neural system (NS), Cellular system (CS) and Immune system (IS) and other on Non human bodies discussing the social insects swarm examples i.e. honey bees, an ant, the wasp and the termites work strategies for practical energy optimization problems. Some limits of the current study that will be further investigated in a future work.

In the genetic system gene structure concept is imported to design wireless sensor networks. By intensively replication of Gene Regulatory Network (GRN) concept can be design more adaptative, robust and dynamic network environments which conserve optimum energy in sensor networks. The nerves system intelligence can be extended for training the sensor nodes for intelligent energy management dynamically. The cellular automation system introduced used for Coordination and control in massively distributed systems; programming of network-centric operating sensor and actor networks It can be used to solve more complexities using advance algorithmic approach in distributed computation for wireless sensor network using local information. Immune system can be replicated for method anomaly and misbehaviour detection. The idea can be extended for the multi-objective minimum energy network connectivity problems for sensor networks.

During the swarm preparation, scout bees will simply find a nearby location for the swarm to cluster. This intermediate stop is not for permanent habitation and will normally leave within three days to a suitable location. It is from this temporary location that the cluster will determine the final nest site based on the level of excitement of the dances of the scout bees. In real world scenario of swarm domain, introduction of mechanisms to limit the number of migratory spices to decrease the network load; further work can be extended to study sensitively the lifecycle scenario for the scouts who is responsible to search the new destination: practically, the servers can also crash and some scouts can also be lost, it should be possible to regenerate them; further investigation on the scout memory to decide the number of sites 
to be remembered and finally the refinement of model the quality throughput in a dynamic environment.

The research at physical layers can be extended for the components such as a power unit, storage unit, sensing unit, processing unit, of sensor nodes that provides an opportunities for optimization using the tuneable parameters like processor voltage and frequency, sensing frequency, duty cycle, etc., whose values can be specialized to meet varying application requirements. The effective optimization can be possible with the neural system and the swarm - an ant's analogy. The energy optimization issues can effectively handle using human immune system and the Swarms like wasps and ants concepts by tuning MAC layer protocol parameters i.e. channel access schedule, message size, duty cycle, and receiver power-off, etc at data link level. The energy efficiency, robustness and scalability can be achieved through data dissemination and routing at network layer. The biological theme of cellular automation \& genetics system and the Swarm - honey bees can give effective solution for energy conservation. The optimization can be achieved like adjustment in transmission power, routing strategies through genetics \& swarms analogy effectively. The energy optimization with effective event handling in operating system can be achieved through Cellular automation and the swarm concepts.

There are many ways we can extend this work. We plan to modify the current conventional approaches with issue of optimum energy using topology control theme. In real networks nodes structure is not always have static state, and even if they were, some routing schemes will want to choose relay nodes to meet QoS requirements. If nodes are chosen around the optimum distance with some probability, then the optimum transmit energy would likely change. Another area of our future work is to test this analysis on actual hardware (e.g., motes) and evaluate the results. There are many other examples of interest as a nature inspired analogy can be used to solve complex problem of application in sensor networks, In biological swarming, Insects like cockroaches, locusts, birds migration, marine life like fish migration, krill, copepods, algal blooms and other animals like bacteria and quadrupeds are also have typical smart behaviour, can be inherited to solve complex problem efficiently.

\section{Conclusions}

The paper has introduced the nature science inspired methods for controlling and managing wireless sensor networks. To establish design of the networking environment, we should have scientific basis. The importance of the 
interdisciplinary approach like natural science can be used for establishing the new environment to networking engineering problems.

The future network demands great dynamism in the networking environment. It will be based on dynamics of the system which will be scalable, self organized and adaptive. Fortunately, the natural processes essentially have such characteristics and that's the reason why the naturally inspired approach is so important. To learn from the natural science is not just an analogy, but a scientific basis of the interdisciplinary science/engineering approach, which is now widely recognized as a source of the innovation in the next generation technology.

The applications of natural science process have been further extended to various areas in order to manage problems, production and giving more accurate solutions dynamically. The paper will provides a reference to researchers, practitioners, and students in both artificial intelligence and engineering communities, forming a foundation for the development of the Nature inspired solution for real world problems of energy optimization in wireless sensor networks.

\section{References}

[1] Azade Nazi, Mayank Raj, Mario Di Francesco, PreetamGhosh, Sajal K. Das, "Robust Deployment of Wireless Sensor Networks Using Gene Regulatory Networks". ICDCN 2013.

[2] H. He, Z. Zhu, and E. Mäkinen, "A neural network model to minimize the connected dominating set for self-configuration of wireless sensor networks," IEEE Transactions on Neural Networks, vol. 20, no. 6, pp. 973-982, 2009.

[3] J. Podpora, L. Reznik, and G. Von Pless, "Intelligent realtime adaptation for power efficiency in sensor networks,'IEEE Sensors Journal, vol. 8, no. 12, pp. 2066-2073, 2008.

[4] A. I. Moustapha and R. R. Selmic, "Wireless sensor network modeling using modified recurrent neural networks: application to fault detection," IEEE Transactions on Instrumentation and Measurement, vol. 57, no. 5, pp. 981-988, 2008.

[5] Stavros Athanassopoulos, Christos Kaklamans, PanagiotaKatsikouli, EviPapaioannou, "Cellular automata for topology control in Wireless Sensor Networks" MELECON IEEE Mediterranean Electrotechnical Conference - MELECON, pp. 212-215, 2012

[6] Xingjia Lu, Yongsheng Ding, KuangrongHao, "Adaptive design optimization of wireless sensor networks using Artificial Immune Algorithms", 2008 IEEE Congress on Evolutionary Computation.

[7] Automata Carlos A. Narv'aez, Rodrigo Lopez, Andr'es Tovar, Diego Garz'on Topology "Synthesis of Compliant Mechanisms using Cellular", EngOpt 2008 - International Conference on Engineering Optimization Rio de Janeiro, Brazil, June 08. 
[8] ArashNikdel, S. MahdiJameii, MohamadSabour, "AISAC: A Novel Artificial Immune System-based Area Coverage Protocol for WSNs", The 2011 International Conference on Wireless Networks, ISBN \#: 1-60132-187-2.

[9] B. K. Panigrahi, Y. Shi, and M. H. Lim (eds.): Handbook of Swarm Intelligence. Series: Adaptation, Learning, and Optimization, Vol 7, Springer-Verlag Berlin Heidelberg, 2011. ISBN 978-3-642-17389-9.

[10] C. Blum and D. Merkle (eds.).Swarm Intelligence - Introduction and Applications. Natural Computing. Springer, Berlin, 2008.

[11] G. Beni and J. Wang, Swarm intelligence in cellular robotic s ystems. In NATO Advanced Workshop on Robots and Biological Systems, Il Ciocco, Tuscany, Italy, 1989.

[12] M. Dorigo, E. Bonabeau, and G. Theraulaz, 'Ant algorithms and stigmergy, Future Generation'. Computation. Syst., Vol. 16, No. 8, pp. 851-871, 2000.

[13] ShabanaMehfuz and M. N. Doja, "Swarm Intelligent Power-Aware Detection of Unauthorized and Compromised Nodes in MANETs", Journal of Artificial Evolution and Applications Volume 2008, Article ID 236803,16pages.

[14] Eric Bonabeau, Marco Dorigo, and Guy Theraulaz, Swarm Intelligence:From Natural to Artificial Systems Oxford University Press, 1999.

[15] Dicke E, Byde A, Cliff D, Layzell P (2004). 'An ant-inspired technique for storage area network design'.In A. J. Ispeert, M. Murata \& N. Wakamiya. Proceedings of Biologically Inspired Approaches to Advanced Information Technology: First International Workshop, BioADIT 2004 LNCS 3141. pp. 364-379.

[16] KarimaAksa, Mohammed Benmohammed, "A Comparison Between Geometric and Bio-Inspired Algorithms for Solving Routing Problem in Wireless Sensor Network", International Journal of Networks and Communications 2012, 2(3): 27-32 DOI: 10.5923/j.ijnc.20120203.02.

[17] Z. Pooranian, A. Barati and A. Movaghar, "Queen-bee Algorithm for Energy Efficient Clusters in Wireless Sensor Networks", World Academy of Science, Engineering and Technology 492011.

[18] Cicirello V. A. and Smith S. F., 'Wasp-like Agents for Distributed Factory Coordination'. J. of Autonomous Agents and Multi-Agent Systems, 8(3): 237-266.

[19] AdamuMurtalaZungeru, Li-MinnAng, KahPhooiSeng 'Performance of Termite-Hill Routing Algorithm on Sink Mobility in Wireless Sensor Networks', SPRINGER Third International Conference, ICSI 2012, Shenzhen, China, June 17-20, 2012 Proceedings, Part II, pp 334-343,2012.

[20] Yang Yang'XuesongQiu, LuomingMeng, KepingLong, 'Task coalition formation and self-adjustment in the wireless sensor networks', Wiley International Journal of Communication Systems, NOV 2012.

[21] ArslanMunir and Ann Gordon-Ross, 'Sustainable Wireless Sensor Networks', book chapter 13, ISBN 978-953-307-297-5, published: Dec, 2010.

[22] Matthew Holland, Tianqi Wang, BulentTavli, AlirezaSeyedi and Wendi Heinzelman, 'Optimizing Physical Layer Parameters for Wireless Sensor Networks,' under revision, IEEE Transactions on Sensor Networks, 2009.

[23] Lu, C., Blum, B., Abdelzaher, T., Stankovic, J. \& He, T., "RAP: A Real-Time Communication Architecture for Large-Scale Wireless Sensor Networks", Real-Time and Embedded Technology and Applications Symposium (RT AS)'02, San Jose, California 


\section{Sanjeev Wagh and Ramjee Prasad}

[24] He, T., Stankovic, J., Lu, C. \&Abdelzaher, T., "SPEED: A Stateless Protocol for Real-time Communication in Sensor Networks", Proc. of International Conference on Distributed Computing Systems (ICDCS)'03, IEEE, Providence, Rhode Island.

[25] Heinzelman, W., Chandrakasan, A. \&Balakrishnan, H. "Energy-Efficient Communication Protocols for Wireless Microsensor Networks", Hawaiian International Conference on System Sciences. 2000.

[26] TinyOS URL: http://www.tinyos.net/ (2010)

[27] Sinha, A. \&Chandrakasan, A. "Operating System and Algorithmic Techniques for Energy Scalable Wireless Sensor Networks", Proc. of International Conference on Mobile Data Management, Hong Kong, 2001 pp. 199-209.

[28] Abrach, H. \& et al. (2003). MANTIS: System Support for Multimodal Networks of In-Situ Sensors, Proc. of Workshop on Wireless Sensor Networks and Applications (WSNA)'03, San Diego, California, pp. 50-59.

[29] Masayuki Murata, "Biologically-Inspired Network Architecture for Future Networks", IWNC 2009, PICT 2, pp. 34-41, 2010, Springer 2010.

[30] https://www.hsdl.org/?home 